\title{
Modal propagation characteristics of radially stratified and D-shaped metallic optical fibers
}

\author{
Charles Y. H. Tsao, David N. Payne, and Luksun Li
}

\begin{abstract}
The eigenvalue equation is formulated for a general three-layered radially stratified metallic optical fiber waveguide and solved numerically using the zoom search method. The result is shown to be applicable to the common D-shaped fiber, which bears no similarity to a concentric stratum but may be converted as such through the Mobius conformal representation. The theoretical prediction agrees well with our experimental measurements, and the method should be proved valuable for optimizing metallic fiber design relationships.
\end{abstract}

\section{Introduction}

Intentional inclusion of a metal layer near or along a light propagation path has become increasingly fashionable in optical fiber designs and integrated optics. ${ }^{1-17}$ It produces highly desirable asymmetric effects on TM and TE electromagnetic waves, which are inspired by numerous optical devices; and as a result the polarizer, for example, with an extinction ratio as high as $60 \mathrm{~dB}$ is recently reported. ${ }^{1-3}$ To improve further the performance of these devices the propagation characteristics of guided waves must be thoroughly understood so their design relations may be optimized.

Regarding the analysis treatment, the prevailing technique is to model metallic waveguides as a type of stratified planar structure, otherwise known as the slab model, which poses the least daunting mathematical complications and with which various waveguide problems can be solved analytically and computationally. ${ }^{11-14}$

Too often this 1-D slab model is taken for granted and referred to without questioning how much similarity to a slab the specific waveguide geometry bears. Surely there must be a limit beyond which the credibility (or lack of it) of a simplistic slab needs to be reexamined. Whenever necessary an alternative should be tried.

The primary objective of this paper is dedicated to this purpose. In particular, using the vector field anal-

The authors are with University of Southampton, Department of Electronics \& Computer Science, Optical Fiber Group, Southampton, S01 9NH, U.K.

Received 17 February 1988.

0003-6935/89/030588-07\$02.00/0.

(C) 1989 Optical Society of America. ysis this paper provides a set of eigenvalue equations (EVEs) for various modes for a three-layered radially stratified metallic waveguide which is theoretically accurate and applicable to both weak or strong fiber waveguides and with which the glass/metal fibers structured as such a genuine stratum may be characterized. For the other large class of so-called Dshaped fiber-a circular dielectric core faces against an additional plane-limited metal clad-the application of the suggested model does not seem to be so straightforward unless the geometry is converted to be radially stratified using the well-known conformal mapping method. This procedure is demonstrated in some detail, and the result is compared with the experimental data.

\section{Radially Stratifled Metallic Fiber Waveguide}

Figure 1 shows the cross section of a typical threelayered stratified fiber which has a core with radius $r_{1}$ and surrounding dielectric and metallic cladding of radius $r_{2}$. The refractive indices for these three regions are, respectively, $n_{1}, n_{2}$, and $n_{3}$, where $n_{1}$ is the core index, $n_{2}$ is the cladding index, both real but $n_{1}>$ $n_{2}, n_{3}$ is the complex refractive index for the metal, namely, $n_{3}=n_{3 r}+j n_{3} i$. (The parameter $n_{3} r$ alone is sometimes referred to as the index of refraction and $n_{3} i$ the extinction coefficient.) Our task now is to establish the electromagnetic fields first and then to formulate analytic eigenvalue equations for TE and TM modes separately because they are no longer degenerate.

If we assume that electromagnetic waves are propagating along the $z$ axis in a cylindrical coordinate, the time-distance factor $\exp [-j(\omega t-\beta z)]$ must be included in the field description. Here $\omega$ is the light circular frequency and $\beta=\beta_{r}+j \beta_{i}$ is the propagation constant which is necessarily complex because of the complex 


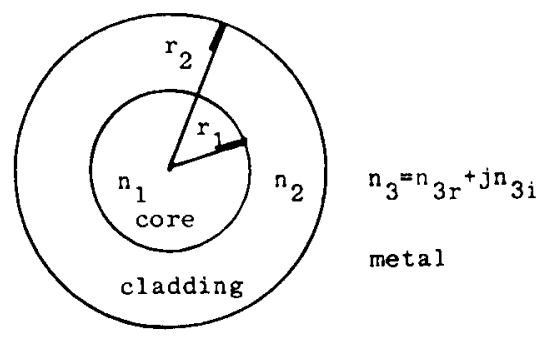

Fig. 1. Typical three-layered radially stratified fiber.

$n_{3}$. Using Debye potentials $z_{0} \Psi \exp (j \beta z)$ and $z_{0} \Phi$ $\exp (j \beta z)$, associated with electric and magnetic fields $\mathbf{E}=\mathbf{e} \exp (j \beta z)$ and $\mathbf{H}=\mathbf{h} \exp (j \beta z)$, time harmonic electromagnetic fields in the above radially stratified waveguide are well known as ${ }^{18}$

$$
\begin{aligned}
\mathbf{e}= & (\partial \Psi / r \partial \phi-\beta \partial \Phi / \omega \epsilon \partial r) \mathbf{r}_{0}+(-\partial \Psi / \partial r-\beta \partial \Phi / \omega \epsilon r \partial \phi) \phi_{0} \\
& -\left(k^{2} n^{2}-\beta^{2}\right) \Phi \mathbf{z}_{0} / j \omega \epsilon, \\
\mathbf{h}= & (\partial \Phi / r \partial \phi+\beta \partial \Psi / \omega \mu \partial r) \mathbf{r}_{0}+(-\partial \Phi / \partial r+\beta \partial \Psi / \omega \mu r \partial \phi) \phi_{0} \\
& +\left(k^{2} n^{2}-\beta^{2}\right) \Psi \mathbf{z}_{0} / j \omega \mu .
\end{aligned}
$$

Here $\mathbf{r}_{0}, \phi_{0}$, and $\mathbf{z}_{0}$ are the unit radial, tangential, and axial vectors, respectively; $\mu$ and $\epsilon$ are the magnetic permeability and electric permittivity which is obviously region dependent.

Formidable as Eqs. (1) and (2) may look at first, they offer a straightforward way to depict fields for any modes, because Debye potentials satisfy the following Helmholtz equations,

$$
\begin{aligned}
& {\left[\Delta_{t}+\left(k^{2} n_{i}^{2}-\beta^{2}\right)\right] \Psi_{i}=0,} \\
& {\left[\Delta_{t}+\left(k^{2} n_{i}^{2}-\beta^{2}\right)\right] \Phi_{i}=0}
\end{aligned}
$$

(with subscription $i$ denoting the layer), of which the solution is already known. Having obtained $\Psi$ and $\Phi$, simple substitution into Eqs. (1) and (2) enables one to derive field components and, therefore, the EVE as one wishes. The status quo of this approach requires no special mathematical skills while the formalism remains accurate and sufficiently concise. Here we shall take the TE mode as an example to illustrate how this can be done.

Introducing $f_{\nu}(\phi)=\exp (j \nu \phi)$ ( $\nu$ is an integer), $U_{1}^{2}=$ $r_{1}^{2}\left(k^{2} n_{1}^{2}-\beta^{2}\right), \quad U_{2}^{2}=r_{1}^{2}\left(k^{2} n_{2}^{2}-\beta^{2}\right)$, and $W_{3}^{2}=r_{2}^{2}\left(\beta^{2}-k^{2} n_{3}^{2}\right)$, the $\Psi_{i}$ terms are easily envisaged to be

$$
\begin{aligned}
& \Psi_{1}=A_{1} J_{\nu}\left(U_{1} r / r_{1}\right) f_{\nu}(\nu \phi), \\
& \Psi_{2}=\left[A_{2} J_{\nu}\left(U_{2} r / r_{1}\right)+B_{2} Y_{\nu}\left(U_{2} r / r_{1}\right)\right] f_{\nu}(\nu \phi), \\
& \Psi_{3}=A_{3} K_{\nu}\left(W_{3} r / r_{2}\right) f_{\nu}(\nu \phi),
\end{aligned}
$$

where $J_{\nu}, Y_{\nu}$, and $K_{\nu}$ are Bessel, Neumann (or Weber), and Hankel functions, respectively, and $U_{1}, U_{2}$, and $W_{3}$ are the layer phase parameters. [Note that $U_{2}$ may be replaced by $W_{2}\left(U_{2}=j W_{2}\right)$, and so $J$ and $Y$ in Eq. (5b) may be replaced by $I$ (Basset function) and $K$, but the final EVEs and their solutions will be identical.] For a TE mode, $\Phi_{i}=0$ may be assumed, which, together with Eqs. (1), (2), and (5), would give rise to

$$
\begin{aligned}
& e_{\phi 1}=-A_{1}\left(U_{1} / r_{1}\right) J_{\nu}\left(U_{1} r / r_{1}\right), \\
& e_{\phi 2}=-\left(U_{2} / r_{1}\right)\left[A_{2} J_{\nu}\left(U_{2} r / r_{1}\right)+B_{2} Y_{\nu}^{\prime}\left(U_{2} r / r_{1}\right)\right], \\
& e_{\phi 3}=-A_{3}\left(W_{3} / r_{2}\right) K_{\nu}\left(W_{3} r / r_{2}\right), \\
& h_{z 1}=-\left(U_{1}^{2} / r_{1}^{2} j \omega \mu\right) A_{1} J_{\nu}\left(U_{1} r / r_{1}\right), \\
& h_{z 2}=-\left(U_{2}^{2} / r_{1}^{2} j \omega \mu\right)\left[A_{2} J_{\nu}\left(U_{2} r / r_{1}\right)+B_{2} Y_{\nu}\left(U_{2} r / r_{1}\right)\right], \\
& h_{z 3}=\left(W_{3}^{2} / r_{2}^{2} j \omega \mu\right) A_{3} K_{\nu}\left(W_{3} r / r_{2}\right) .
\end{aligned}
$$

Four coefficients $A_{1}, A_{2}, A_{3}$, and $B_{2}$ are arbitrarily selected to scale the field, yet they are not totally independent of each other in a sense that they may be defaulted to produce the EVE through boundary continuities such as those involving $e_{\phi}$ and $h_{z}$. For the structure depicted in Fig. 1 there are only two boundaries dividing the core, dielectric, and metal cladding, and application of the continuity of transverse fields yields a $4 \times 4$ matrix of which the determinant must be zero to ensure this coefficient independency. So we have

TE mode:

$$
\begin{array}{r}
{\left[\begin{array}{l}
\left.J_{\nu}\left(U_{1}\right) p_{\nu} / U_{1} J_{\nu}\left(U_{1}\right)-q_{\nu} / U_{2}\right]\left[K_{\nu}\left(W_{3}\right) p_{\nu} / W_{3} K_{\nu}\left(W_{3}\right)\right. \\
\left.+r_{\nu} / a U_{2}\right]-\left(2 / \pi a U_{2}^{2}\right)^{2}=0,
\end{array}\right.}
\end{array}
$$

where $p_{\nu}=J_{\nu}\left(a U_{2}\right) Y_{\nu}\left(U_{2}\right)-J_{\nu}\left(U_{2}\right) Y_{\nu}\left(a U_{2}\right), q_{v}=$ $J_{\nu}\left(a U_{2}\right) Y_{\nu}\left(U_{2}\right)-J_{\nu}\left(U_{2}\right) Y_{\nu}\left(a U_{2}\right)$, and $r_{\nu}=J_{\nu}\left(a U_{2}\right)$ $Y_{\nu}\left(U_{2}\right)-J_{\nu}\left(U_{2}\right) Y_{\nu}\left(a U_{2}\right)$ are the cross products of Bessel and Neumann functions ${ }^{19}$; and $a=r_{2} / r_{1}$ is the ratio of the two radii. This routine is repeatable for the TM mode for which $\Psi_{i}=0$ may be adopted, and the final EVE takes the form of Eq. (8).

TM mode:

$$
\begin{aligned}
& {\left[J_{\nu}^{\prime}\left(U_{1}\right) p_{\nu} / U_{1} J_{\nu}\left(U_{1}\right)-n_{2}^{2} q_{\nu} / n_{1}^{2} U_{2}\right]\left[K_{\nu}\left(W_{3}\right) p_{\nu} / W_{3} K_{\nu}\left(W_{3}\right)\right.} \\
& \left.+n_{2}^{2} r_{\nu} / n_{3}^{2} a U_{2}\right]-\left(2 n_{2}^{2} / \pi n_{1} n_{3} a U_{2}^{2}\right)^{2}=0 .
\end{aligned}
$$

Here $n_{1}=n_{2}$ and $\nu=0$ may be assumed if only weakly guided $\mathrm{TM}_{0}$ or $\mathrm{TE}_{0}$ modes are of interest. In general, it is Eqs. (7) and (8) which characterize precisely all transverse modes.

The presence of both $\Phi_{i}$ and $\Psi_{i}$ in Eqs. (1) and (2) leads to a similar EVE for a hybrid HE/EH mode; details are described in Ref. 17.

\section{Comparlson with the Two-Layered Stratifled Wavegulde}

A three-layered stratified waveguide can easily be degraded as a two-layered one. For example, in Fig. 1 where the dielectric cladding layer is so thin that $r_{2}=r_{1}$ holds, the structure could represent a two-layered glass/metal $\left(n_{1}-n_{3}\right)$ or hollow metallic $\left(n_{1}=1\right)$ waveguide. The other extreme is to thicken the dielectric cladding to such an extent that the fiber is apparently of the step-index profile $\left(n_{1}-n_{2}\right)$. Equations (7) and (8) have to be reducible to be in more familiar forms.

For the purpose of examining the step-index profile we ought to redefine the phase parameter $W_{2}^{2}=r_{1}^{2}\left(\beta^{2}-\right.$ $\left.k^{2} n^{2}\right)$ or $U_{2}=j W_{2}$. In view of $J_{\nu}\left(j W_{2}\right)=\exp (j \nu \pi /$ 2) $I_{\nu}\left(W_{2}\right), Y_{\nu}\left(j W_{2}\right)=\exp [j(\nu+1) \pi / 2] I_{\nu}\left(W_{2}\right)-(2 / \pi)$ 
$\exp (-j \nu \pi / 2) K_{\nu}\left(W_{2}\right)$ (provided that $-\pi<\arg W_{2}<$ $\pi / 2),{ }^{19}$ etc., the cross products are alternatively defined as

$$
\begin{aligned}
p_{\nu}= & -(2 / \pi)\left[I_{\nu}\left(a W_{2}\right) K_{v}\left(W_{2}\right)-I_{\nu}\left(W_{2}\right) K_{\nu}\left(a W_{2}\right)\right], \\
q_{\nu}= & j(2 / \pi)\left\{\nu\left[I_{\nu}\left(a W_{2}\right) K_{\nu}\left(W_{2}\right)-I_{\nu}\left(W_{2}\right) K_{\nu}\left(a W_{2}\right)\right] / W_{2}\right. \\
& \left.-\left[I_{\nu}\left(a W_{2}\right) K_{\nu+1}\left(W_{2}\right)+I_{\nu+1}\left(W_{2}\right) K_{\nu}\left(W_{2}\right)\right]\right\}, \\
r_{\nu}= & j(2 / \pi)\left\{\nu\left[I_{\nu}\left(a W_{2}\right) K_{\nu}\left(W_{2}\right)-I_{\nu}\left(W_{2}\right) K_{\nu}\left(a W_{2}\right)\right] / a W_{2}\right. \\
& \left.+\left[I_{v}\left(W_{2}\right) K_{v+1}\left(a W_{2}\right)+I_{v+1}\left(a W_{2}\right) K_{\nu}\left(W_{2}\right)\right]\right\} .
\end{aligned}
$$

Here $W_{2}$ may be simply denoted as $W$ if only two layers are considered. An infinitely large $a$ means an $I_{\nu}\left(a W_{2}\right)$ dominance in Eqs. (9), i.e., $p_{v} \approx-(2 / \pi) I_{\nu}\left(a W_{2}\right) K_{\nu}\left(W_{2}\right)$, $q_{\nu} \approx j(2 / \pi) I_{\nu}\left(a W_{2}\right) K_{\nu}^{\prime}\left(W_{2}\right)$, and $r_{\nu} \approx j(2 / \pi)$ $I_{\nu}\left(a W_{2}\right) K_{\nu}\left(W_{2}\right)$, which in turn makes the last terms in Eqs. (7) and (8) absolutely negligible. Thus we may deduce

TE mode:

$$
J_{\nu}^{\prime}\left(U_{1}\right) / U_{1} J_{\nu}\left(U_{1}\right)-q_{\nu}^{\prime} / j W_{2} p_{v}=0
$$

TM mode:

$$
J_{\nu}\left(U_{1}\right) / U_{1} J_{\nu}\left(U_{1}\right)-n_{2}^{2} q_{\nu} / n_{1}^{2} j W_{2} p_{\nu}=0 .
$$

Here $q_{\nu} / p_{\nu} \approx-j K_{\nu}\left(W_{2}\right) / K_{\nu}\left(W_{2}\right)$. By letting $\nu=0$, Eqs. (10) and (11) are exactly the EVE for the step-profiled all-dielectric fiber. ${ }^{20}$

As far as the hollow metallic waveguide, ${ }^{21}$ or its like, is concerned, the dielectric cladding may be treated as an extension of the core, i.e., $n_{2}=n_{1}, r_{2}=r_{1}+\delta r_{1}$, and $\delta$ $\rightarrow 0$. It follows that $a=1+\delta, p_{\nu}=-2 \delta / \pi, q_{\nu}=2 / \pi U_{1}$, and $r_{v}=-2 / \pi U_{1}$, since $U_{2}=U_{1}$. All these relations explain why Eqs. (7) and (8) are reduced to Eqs. (12) and (13), which is nothing but the hollow metallic waveguide $\operatorname{EVE}\left(n_{1}=1\right):{ }^{21}$

TE mode:

$$
J_{\nu}\left(U_{1}\right) / U_{1} J_{\nu}\left(U_{1}\right)+K_{\nu}\left(W_{3}\right) / W_{3} K_{\nu}\left(W_{3}\right)=0,
$$

TM mode:

$$
J_{\nu}^{\prime}\left(U_{1}\right) / U_{1} J_{v}\left(U_{1}\right)+n_{3}^{2} K_{\nu}\left(W_{3}\right) / n_{1}^{2} W_{3} K_{\nu}\left(W_{3}\right)=0 .
$$

The fact that the EVE of a three-layered waveguide is more general and reducible to cover various two-layered ones is no guide to feelings that it indiscriminately applies to all metallic fibers. Extreme care is required when applied because the result proposed here is, strictly speaking, irrelevant to some fibers unless they are genuinely three-layered concentric or convertible to be such.

\section{D-Shaped Metallic Flbers}

Some metallic fibers are D-shaped where the metal sector forms a letter $\mathrm{D}$ while the core remains circular [Fig. 2(a)].1-3 Experience shows that the performance of this fiber does not render it to be $\mathrm{D}$-shape dependent as long as it is reasonably thick. Using a slab waveguide model the criterion of the so-called thick metal has been investigated for most common metals, but as a rule of thumb a few microns are often sufficient. ${ }^{14}$
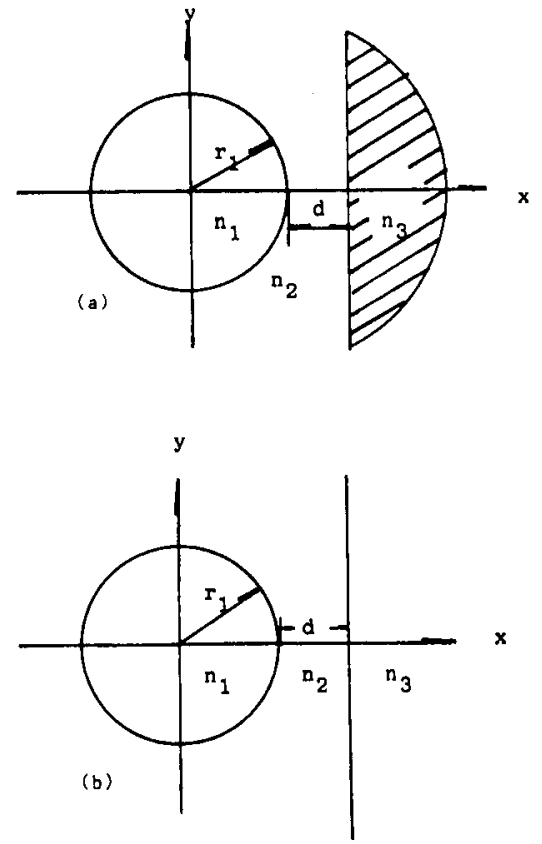

Fig. 2. (a) Fiber cross section of a typical D-shaped fiber. (b) The D-shaped fiber is modeled as having a circular core and an additional plane-limited metal cladding.

Although this slab model is different from the Dshaped fiber, electromagnetic waves should not penetrate to a great depth in metal in either case due to the skin effects; and so most D-shaped fibers may be accordingly modeled as having a circular core and a planar metal cladding [see Fig. 2(b)]. The problem now is to seek a simple solution for this ill-fitted waveguide geometry.

The answer may lie in the conformal representation (or mapping) through which the ill-fitted irregular geometry on the $z$-plane-a circle accompanied by a straight line in this case-may be regulated to be all straight lines or concentric circles on a new $\zeta$-plane so that the difficulty in finding a solution may be palliated. This mapping technique could be plausible if the following problems can be solved.

First, although the geometry is seemingly simplified on the new plane, the Helmholtz wave equation could easily become harder to solve because it is now in ${ }^{22}$

$$
\left[\Delta_{t}+\left(k^{2} n^{2}-\beta^{2}\right) / /\left.\zeta^{\prime}(z)\right|^{2}\right](\Psi \text { or } \Phi)=0 .
$$

Here $\zeta(z)$ is the conformal representation, $\zeta^{\prime}(z)=d \zeta / d z$ remains continuous and analytic defined in the Cauchy-Riemann sense, and $\zeta \neq 0$ supposedly holds for any $z$. It is seen that the regulation of the geometry is achieved at the price of complicating the wave equation. As it may be clearer later that $\left|\zeta^{\prime}(z)\right|^{2}$ can be too tricky, Eq. (14) may not lend itself to an easy solution.

Second, we must decide what our mapping function is. But how? The answer is to consult the literature (e.g., Refs. 22-24) hoping to come across something relevant. A little caution is needed, however, since we may pick up many options, and chances are some functions may be more suitable than others. For example, the Mobius representation ${ }^{23,24} \zeta(z)=r_{2}\left(z-x_{1}\right) /\left(z-x_{2}\right)$ 
may be considered appropriate for the problem in Fig. 2 , because it requires no edge effect correction and still converts two discrete circles to a pair of concentric ones (Figs. 3 and 4). Here $r_{2}$ is real, $R_{1}$ and $R_{2}$ are the radii of the $z$-plane, and $x_{1}$ and $x_{2}$ are also real and satisfy

$$
\begin{aligned}
x_{1} \cdot x_{2} & =R_{1}^{2}, \\
\left(R_{1}+R_{2}+d-x_{1}\right) \cdot\left(R_{1}+R_{2}+d-x_{2}\right) & =R_{2}^{2} .
\end{aligned}
$$

So far so good, except Fig. 3 is not yet Fig. 2 unless $R_{2}$ becomes infinitive. Letting it be so gives rise to

$$
\begin{aligned}
& x_{1}=R_{1}+d-\sqrt{R_{1}^{2}+2 R_{1} d}, \\
& x_{2}=r_{2}=R_{1}+d+\sqrt{R_{1}^{2}+2 R_{1} d}, \\
& r_{1}=R_{1},
\end{aligned}
$$

where $d$ is the surface-to-surface separation between the core and metal, $r_{1}$ and $r_{2}$ are, respectively, the inner and outer radius after the mapping, and $x_{1} \ll x_{2}$ is usually true.

How about Eq. (14) then? Differentiating $\zeta$ we get $\left|\zeta^{\prime}(z)\right|^{2}=\left|r_{2}\left(x_{2}-x_{1}\right) /\left(r_{2}-z\right)^{2}\right|^{2}$, which is expectedly, perhaps disappointingly, $z$-dependent.

Let us remind ourselves that all we wish to derive is the detailed field distribution in the waveguide outlined in Fig. 2. But we may have already realized that in spite of the unknown distribution function varying among modes, yet for the lower-order ones the field must be confined within the core. This indicates that only small $|z|$, or small $|\zeta|$ in $|\zeta|$, will more decisively affect the waveguide character. By taking up the point further, let us quote $z=r_{2}\left(\zeta-x_{1}\right) /\left(\zeta-r_{2}\right)$ and rewrite $1 /\left|\zeta^{\prime}\right|=\left(r_{2}-x_{1}\right) /\left|r_{2}\left(1-2 r \cos \phi / r_{2}+r^{2} / r_{2}^{2}\right)\right|$, using $\zeta=r \exp (j \phi)$. Since only small $|\zeta|$ primarily matters, it would not be unthinkable to expand $1 /|\zeta|^{2}$ into a series of power $\left(r / r_{2}\right)$, namely, $1 /\left.\xi^{\prime}\right|^{2}=(1-$ $\left.x_{1} / r_{2}\right)^{2}\left(1+4 r \cos \phi / r_{2}\right)+\ldots$ Now a perturbation method may be invoked for solving Eq. (14). It can be shown that the correction due to the term $4 r \cos \phi / r_{2}$ is absolutely negligible $(<1 \%),{ }^{25}$ and the following zeroorder version of Eq. (14) is adequate:

$$
\left[\Delta_{t}+\left(1-x_{1} / r_{2}\right)^{2}\left(k^{2} n^{2}-\beta^{2}\right)\right](\Psi \text { or } \Phi)=0 .
$$

It can be readily seen that for Eq. (20) the solution in Eq. (5) is applicable if all phase parameters are scaled by a factor of $\left(1-x_{1} / r_{2}\right)$. It is interesting to note that even this minor scale alteration may easily become superfluous in certain circumstances when $x_{1} / r_{2} \ll 1$ is satisfied.

\section{TM and TE Fields in a D-Shaped Fiber}

So far we have only proved one thing, that is, through the Mobius conformal representation the Dfiber is mapped to be radially stratified with its phase parameters scaled on the new plane. It follows that the wave solutions together with their EVEs proposed at the beginning of this paper are applicable if referenced to the $\zeta$-plane. This means that for any given Dshaped fiber design, it is always possible to work out the equivalent concentric structure using the Mobius transformation formulas [Eqs. (17)-(19)]. Having es-

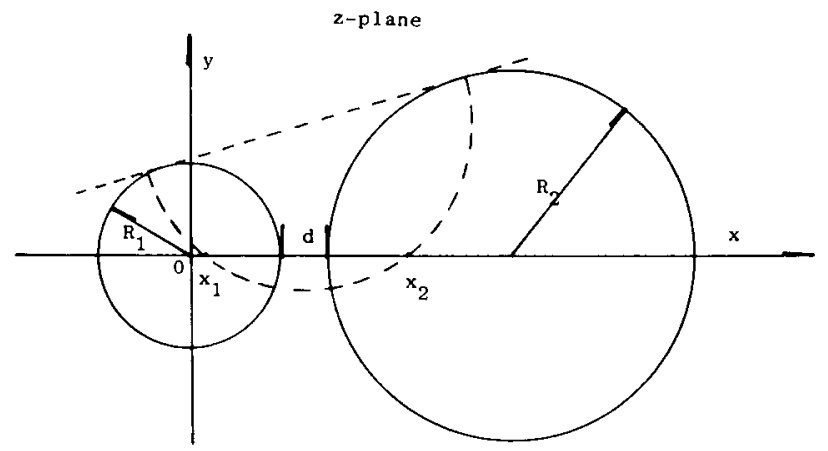

Fig. 3. Two discrete circles on a $z$-plane with $z=x+j y$.

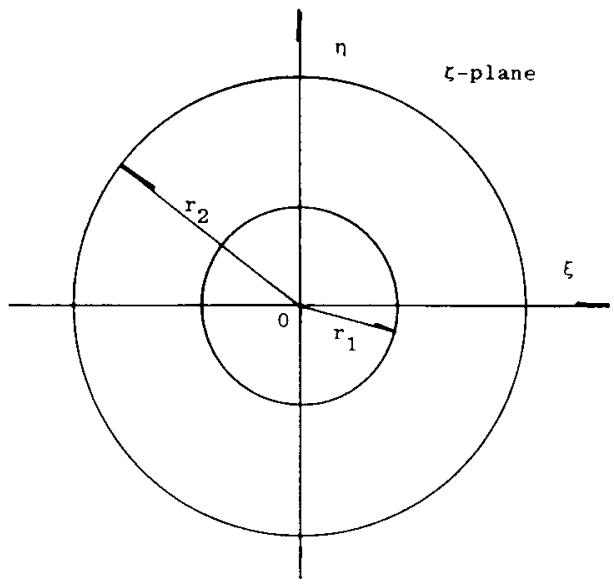

Fig. 4. Two discrete circles on the $z$-plane are mapped to be two concentric ones on the $\zeta$-plane, where $\zeta=\xi+j \eta$.

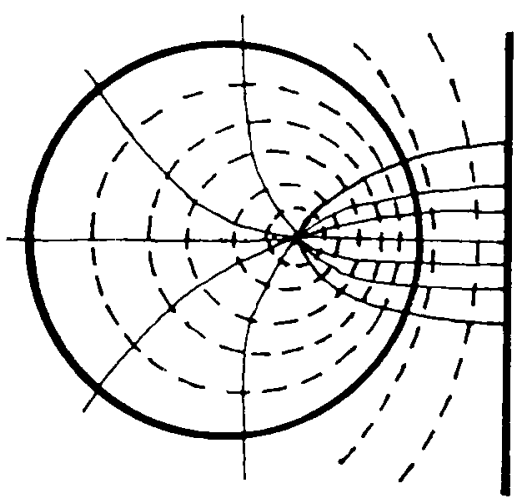

Fig. 5. Electric fields for the TE (broken lines) or TM (solid line) mode in a D-shaped fiber.

tablished the size of these circles, one should be able to form a new phase parameter such as $U_{1}, U_{2}$, and $W_{3}$ to solve the EVEs. Nevertheless, it is worth noting that the electromagnetic field on the original plane bears little similarity with those for bona fide radially stratified fibers (see Fig. 5). 


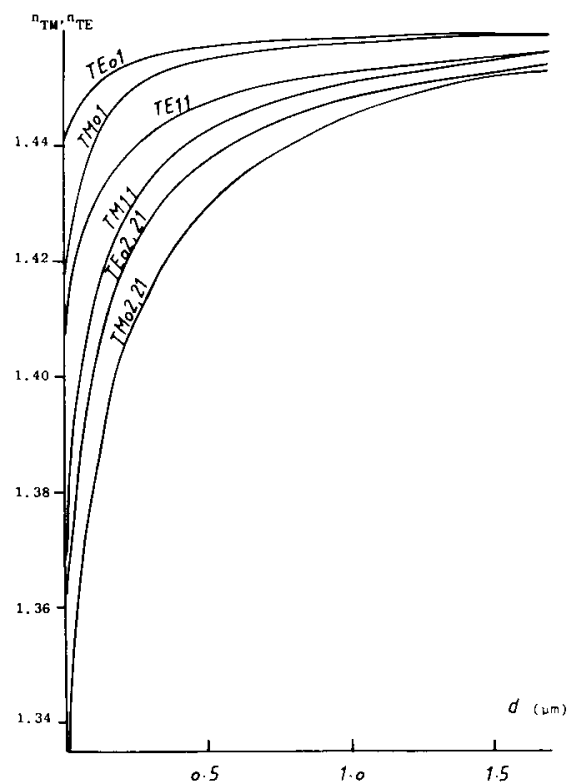

Fig. 6. Equivalent index of the propagation constant for various modes.

\section{Computational Scheme for Solving EVEs}

We wish to calculate attenuations for the various TM and TE modes, and this requires solving transcendental EVEs in Eqs. (7) and (8). For these equations there is no other known method apart from the numerical computation.

From our computer experience it is felt that the zoom search, or numerical zoom analysis, is quite efficient. It shows great advantage in solving a multilayered slab metallic waveguide. ${ }^{14}$ Let us agree that the search routine would have been even more straightforward if none of those arctangent functions were multiple-valued. This is now the case, however, in the radially stratified model.

In a nutshell, what the zoom search requires is to calculate the left-hand side (lfs) of Eqs. (7) and (8) to make sure it is a complex zero for an estimated $\beta^{2}$. If it is, or more precisely, if the magnitude of the lhs is sufficiently small (e.g., $10^{-6}$ as chosen here for our purpose), the solution is found; if not, search again. More specifically, the zoom search encourages one to minimize the magnitude of the lhs while $\operatorname{Re}\left(\beta^{2}\right)$ is scanned downward from $n_{1}^{2}$. As soon as the first minimum is tangible a similar scan is exercized with respect to $\operatorname{Im}\left(\beta^{2}\right)$. To save time and cost, the fine resolution of the first round search must be avoided because of its large area of coverage, although it will progressively improve as the minimization is iterated and the focus of the search is quickly zoomed on a very tiny spot.

It is not a difficult method, despite the fact that we are dealing with a wide range of cylindrical functions of the integer order and complex argument. Nowadays, booming algorithms for computing Bessel and modified Bessel functions are widely available with whatever accuracy is demanded. ${ }^{26-28}$ Based on these algorithms we developed our programs on an IBM PC with relatively weak accuracy (i.e., $10^{-6}$ ), but we believe it is

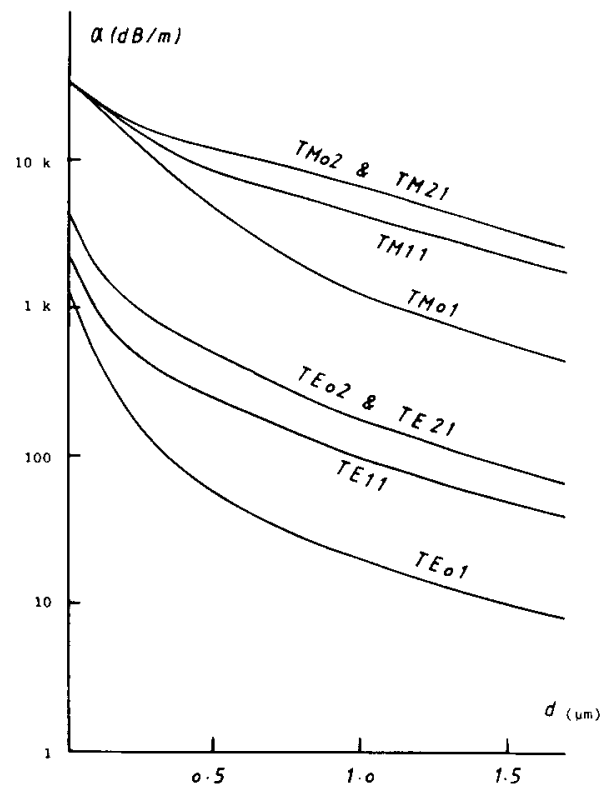

Fig. 7. Attenuations of various modes as a function of the coremetal separation $d$.

probably what we needed at the moment although it can be improved on a PDP-11 and VAX-750.

\section{Numerical Results}

To allay anxieties let us now present some interim results with regard to the general behavior of a radially stratified or D-shaped fiber. More specifically, let us plot the equivalent index and the attenuation rate in terms of decibels for the propagation constant $\beta$ as a function against the separation $d$. The results are shown in Figs. 6 and 7. Here everything else is fixed, e.g., $n_{1}=1.47, n_{2}=1.46, R_{1}=1.5 \mu \mathrm{m}, n_{3 r}=2.245, n_{3 i}=$ 8.85 (gallium). A higher attenuation $\left(\alpha_{\mathrm{TM}}, \alpha_{\mathrm{TE}}\right)$ is observed for a higher-order $\nu$ mode or when the metal is placed closer to the core, meaning more metal effects. This tendency is also evident from the computed equivalent indices $n_{\mathrm{TM}}$ and $n_{\mathrm{TE}}$, which take lower values for higher-order $\nu$ modes and drop steadily while $d$ $\rightarrow 0$. In all cases, $n_{\mathrm{TE}}>n_{\mathrm{TM}}$ and $\alpha_{\mathrm{TM}}>\alpha_{\mathrm{TE}}$ always remain true, which is the key feature of this kind of metallic fiber.

\section{Experimental Results and Comparison with Other Methods}

The model and its solution presented here work very well for real D-shaped fibers when the measured attenuation is compared with predicted values. For example, a high-performance gallium/glass fiber polarizer has recently been made with the parameters chosen as follows: N.A. $=0.20, n_{2}=1.4585, r_{1}=2.0 \mu \mathrm{m}, d=1.0$ $\mu \mathrm{m}$. Measured attenuations $\alpha_{\mathrm{TM}}$ and $\alpha_{\mathrm{TE}}$ at wavelength $\lambda=0.83 \mu \mathrm{m}$ are 1.5 and $0.012 \mathrm{~dB} / \mathrm{cm}$, respectively. The theoretical calculation for this very fiber gives $\alpha_{\mathrm{TM}}=1.57 \mathrm{~dB} / \mathrm{cm}$ and $\alpha_{\mathrm{TE}}=0.0118 \mathrm{~dB} / \mathrm{cm}$. They are indeed rather close to each other.

The D-shaped fiber has been analyzed previously, ${ }_{15}^{15}$ and attenuations of the two so-called $\mathrm{LP}_{01}$ modes are 


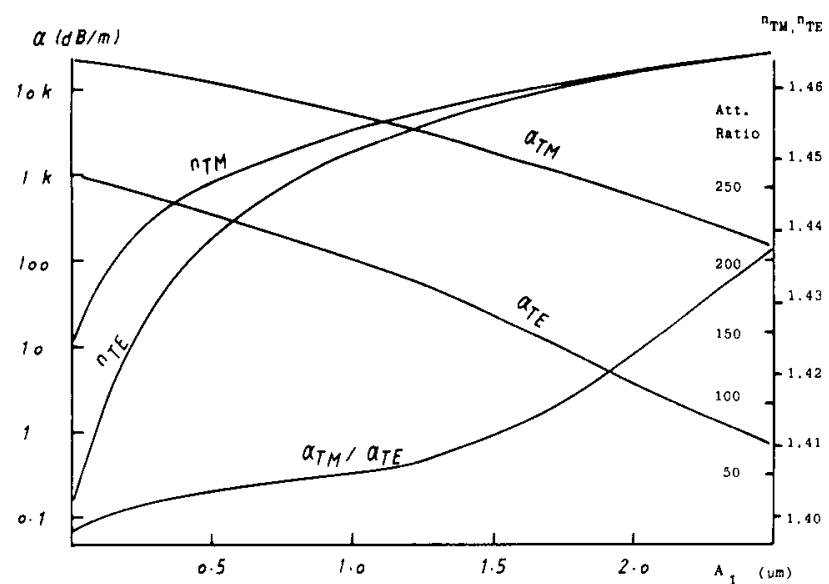

Fig. 8. Waveguide performance in terms of propagation speed and attenuation as a function of the core radius $A_{1}\left(n_{1}=1.47, n_{2}=1.46, d\right.$ $=0.8 \mu \mathrm{m}, \lambda=0.83 \mu \mathrm{m}$, gallium)

observed very close to those for $\left(\alpha_{\mathrm{TM} 01}+\alpha_{\mathrm{TM} 11}\right) / 2$ and $\left(\alpha_{\text {TE01 }}+\alpha_{\text {TE11 }}\right) / 2$, the terms defined and computed through conformal mapping method. (The subscripts 01 and 11 stand for the lowest-order modes for $\nu=0$ and $\nu=1$.) This is interesting, although it might be as important to point out that the $\mathrm{LP}_{01}$ mode makes sense for the two-layered dielectric fibers, which is no longer the mode after the metal is inserted. This new mode is not discussed in Ref. 15; instead, it is approximated by a Bessel-Fourier expansion. In essence this representation differs little from what is regarded as having broken the field down to include various weighted higher-order modes. With the participation of higher-order modes, the attenuation must have been contaminated as well.

Like the slab waveguide model and its recursive EVEs the radially stratified model explored here is analytic and has been proved a favorite for optimizing a fiber design. As an example, the phenomenal waveguide characteristics vs a relative refractive-index difference between the core and cladding $\Delta$ and the core radius $\left(A_{1}\right)$ are illustrated in Figs. 8 and 9 . It is not a surprise to learn from these figures that a smaller $V$ value (a smaller $A_{1}$ or $\Delta$ ) of a single-mode fiber placed in the vicinity of a metal $D$ receives more metal effects simply because the field is more spread out. Nevertheless while $\Delta$ increases further neither the TE nor TM mode will suffer any substantial loss owing to the field confinement which underlies a tuning effect in terms of the attenuation ratio, which has already been observed in a slab metallic waveguide. ${ }^{14}$

\section{Concluding Remarks}

The three-layered radially stratified metallic fiber waveguide has been analytically solved with its EVEs for TE and TM modes formulated. These EVEs are applicable even to common D-shaped fibers since they may be converted as genuine concentric ones. Although these EVEs are transcendental they can be easily computed because of available algorithms for all Bessel, modified Bessel functions, and their cross

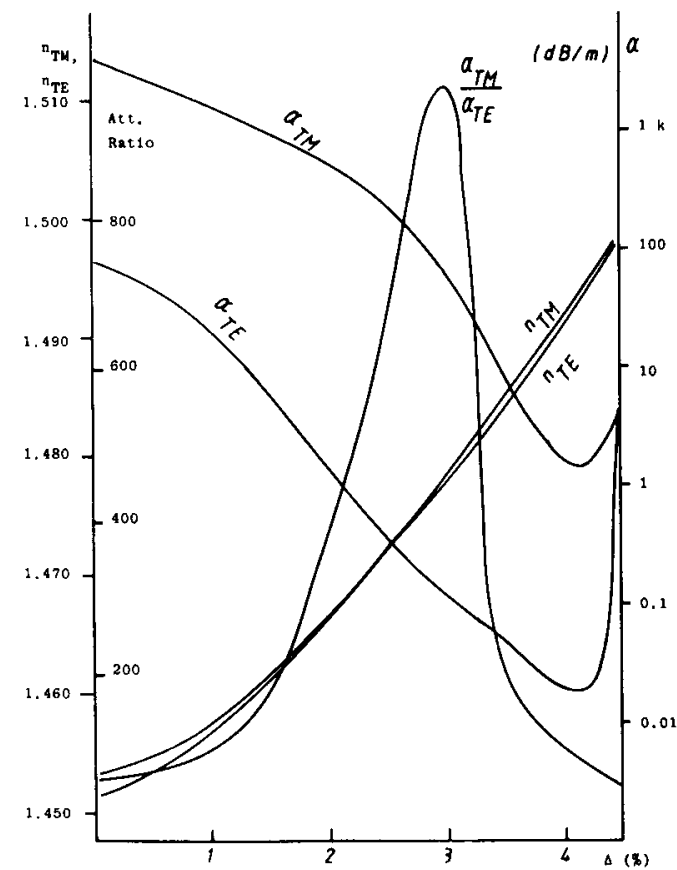

Fig. 9. Waveguide performance as a function of the relative refractive-index difference $\Delta=\left(n_{1}-n_{2}\right) / n_{1},\left(n_{2}=1.4585, d=0.8 \mu \mathrm{m}, r_{1}=\right.$ $1.5 \mu \mathrm{m}$, gallium at $\lambda=0.83 \mu \mathrm{m}$ ).

products. The results in this paper predict measurements satisfactorily, and the method could prove valuable for optimizing metallic fiber designs.

The authors wish to thank W. A. Gambling for his encouragement. Also the authors are grateful to C. D. Hussey, P. St. Russell, S. B. Poole, P. Harris, G. Wylangowski and $M$. Tachibana for discussions and useful comments. This work was supported throughout by the Science and Engineering Council (U.K.), and its help is sincerely acknowledged.

\section{Reterences}

1. L. Li, R. D. Birch, and D. N. Payne, "High-Performance Composite Metal/Glass Fibre Polarisers," in Technical Digest, Twelfth European Conference on Optical Communication, Barcelona (1986), Vol. 1, p. 137-140.

2. L. Li, G. Wylangowski, D. N. Payne, and R. D. Birch, "Low-Cost Metal/Glass Fibre Polarisers Produced," in Technical Digest, Fourth International Conference on Optical Fiber Sensors, Tokyo (1986), p. 163-166.

3. L. Li, G. Wylangowski, D. N. Payne, and R. D. Birch, "Broadband Metal/Glass Single-Mode Fibre Polarisers," Electron. Lett. 22, 1020 (1986).

4. S. C. Rashleigh, "Positive Permittivity Metal Cladding: its Effect on the Modes of Dielectric Optical Waveguides," Appl. Opt. 15, 2804 (1976).

5. K. Thyagarajan, A. N. Kaul, and S. I. Hosain, "Attenuation Characteristics of Single-Mode Metal-Clad Graded-Index Waveguides with a Dielectric Buffer: a Simple and Accurate Numerical Method," Opt. Lett. 11, 479 (1986).

6. Y. Suematsu, M. Hakuta, K. Furuya, K. Chiba, and R. Hasumi, "Fundamental Transverse Electric Field $\left(\mathrm{TE}_{0}\right)$ Mode Selection for Thin-Film Asymmetric Light Guides," Appl. Phys. Lett. 21, 291 (1972).

7. H. F. Mahlein, R. Oberbacher, and W. Rauscher, "An Integrated Optical TE-TM Mode Splitter," Appl. Phys. 7, 15 (1975). 
8. H. F. Mahlein, "Integrated Optical Polarizer," Opt. Commun. 16, 420 (1976).

9. J. Ctyroky, J. Janta, and J. Schrofel, "Thin-Film Polarizer for Optical Waveguides," in Technical Digest, Tenth European Conference on Optical Communication, Stuttgart (1984), p. 4445.

10. J. Ctyroky and H. J. Henning, "Thin Film Polariser for $\mathrm{T}_{\mathrm{i}}: \mathrm{L}_{\mathrm{i}} \mathrm{N}_{\mathrm{b}} \mathrm{O}_{3}$ Waveguides at $\lambda=1.3 \mu \mathrm{m}$," Electron. Lett. 22, 756 (1986).

11. R. Shubert and J. H. Harris, "Optical Guided-Wave Focusing and Diffraction," J. Opt. Soc. Am. 61, 154 (1971).

12. J. N. Polky and G. L. Mitchell, "Metal-Clad Planar Dielectric Waveguide for Integrated Optics," J. Opt. Soc. Am. 64, 274 (1974).

13. Y. Yamamoto, T. Kamiya, and H. Yanai, "Characteristics of Optical Guided Modes in Multilayer Metal/Clad Planar Optical Guide with Low-Index Dielectric Buffer Layer," IEEE. J. Quantum Electron. QE-11, 729 (1975).

14. C.Y. H. Tsao, L. Li, and D. N. Payne, "Propagation Characteristics of Guided Waves in Stratified Metallic Optical Waveguides," Appl. Opt. 27, 1316 (1988).

15. C. Vassallo, "Rigorous Theory for Propagation in Optical Fibres with a Plane-Limited Cladding," in Technical Digest, Twelfth European Conference on Optical Communication, Barcelona (1986), pp. 333-335.

16. S. J. Al-Bader and H. A. Jamid, "Comparison of Absorption Loss in Metal-Clad Optical Waveguides," IEEE. Trans. Microwave Theory Tech. MTT-34, 310 (1986).

17. C. Y. H. Tsao, "Conformal Mapping for D-Shaped Fibres,"
Internal Report, Optical Fibre Group, Electronics Department, U. Southampton (1986).

18. W. R. Smythe and C. Yeh, "Formulas," in American Institute of Physics Handbook, D. E. Gray, Ed. (McGraw-Hill, New York, 1972), Sec. 5.

19. M. Abramowitz and I. A. Stegun, Eds., Handbook of Mathematical Functions (Dover, New York, 1965), Chap. 9.

20. M. J. Adams, An Introduction to Optical Waveguides (Wiley, Chichester, 1981), p. 225.

21. M. Miyagi and S. Kawakami, "Design Theory of DielectricCoated Circular Metallic Waveguides for Infrared Transmission," IEEE/OSA J. Lightwave Technol. LT-2, 116 (1984).

22. P. M. Morse and H. Feshbach, Methods of Theoretical Physics, Vol. 2 (McGraw-Hill, New York, 1953), Chap. 10, p. 1175.

23. R. V. Churchill, Complex Variables and Applications (McGraw-Hill, New York, 1974), Appl. 2, p. 319.

24. H. Kober, Dictionary of Conformal Representations (Dover, New York, 1952).

25. C. Y. H. Tsao, "Vector Wave Equation in Curvilinear Coordinates and its Analytic Solution," Internal Report, Optical Fibre Group, Electronics Department, U. Southampton (1985).

26. J.P. Mason, "Cylindrical Bessel Functions for a Large Range of Complex Arguments," Comput. Phys. Commun. 30, 1 (1983).

27. K. H. Burrel, "Algorithm 484. Evaluation of the Modified Bessel Function $K_{0}(z)$ and $K_{1}(z)$ for Complex Arguments," Commun. ACM 17, 524 (1977).

28. D. J. Sookne, "Bessel Functions $J$ and $I$ of Complex Argument and Integer Order," J. Natl. Bur. Stand. U.S.A. JNBBAU 77B (3 and 4) $73-214$, pp. 111-114, 115-124, 125-132, and 133-136 (1973). 